\title{
ESTIGMA INTERNALIZADO EN PERSONAS CON TRASTORNO MENTAL, RESIDENTES DE HOGAR PROTEGIDO CON REHABILITACIÓN DE BASE COMUNITARIA (PRIMER SEMESTRE DE 2019)
}

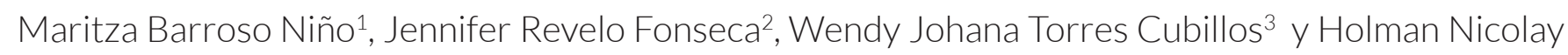
Torres Osorio ${ }^{4}$

\section{Resumen:}

El estigma hace referencia a las actitudes y creencias que conducen a las personas a rechazar, evitar y temer a aquellos a quienes perciben como diferentes; por su parte, el estigma internalizado hace referencia a aquel que es orientado hacia sí mismo.

En este sentido, el objetivo de la presente investigación es determinar el nivel de estigma internalizado en personas con trastorno mental (TM), residentes de un hogar protegido con rehabilitación de base comunitaria, durante el primer semestre de 2019. Para la metodología, se realizó un estudio observacional y descriptivo, de enfoque cuantitativo; además, se utilizaron dos instrumentos: una encuesta de caracterización sociodemográfica de la persona con alteración mental y la aplicación de la escala de autoestigma de la enfermedad Internalized Stigma of Mental IIIness Scale (ISMI) (Tanabe et ál., 2016). Los resultados obtenidos arrojan que las cuatro mediciones permiten verificar que hay un 62,5\% de percepción de estigma y discriminación. A su vez, puntúa la resistencia al estigma en un 70\%, pues se muestra que puede existir una adaptación social en el dispositivo de rehabilitación de base comunitaria que es capaz de contrarrestar el estigma social y evitar que sea internalizarlo en los integrantes de la comunidad. Se concluye que es evidente que existe una alta percepción de alienación, estigma y discriminación, no solo en el ámbito social, que es en el que más se ve este rechazo así como en el mismo aislamiento del paciente, sino a nivel familiar y laboral. Gracias a las practicas comunitarias, la comunidad ha construido mecanismos basados en procesos de pensamiento crítico y entendimiento, que permiten afrontar el estigma social y no lo internalizan.

Palabras clave: trastornos mentales, estigma social, educación en salud, teoría de la mente, esquizofrenia, aislamiento social.

Historial del artículo:

Fecha de recibido: 08-09-2021 - Fecha de aceptado: 07-10-2021

(c) 2021 Fundación Universitaria Juan N. Corpas. FUJNC.

Artículo Open Access bajo la Licencia Creative

Commons Atribución-NoComercial-Compartirlgual 4.0 Internacional.

DOI: https://doi.org/10.26752/revistaparadigmassh.v3iSupl.1.607

\footnotetext{
${ }^{1}$ Enfermera y especialista en perinatología por la Universidad Nacional. Docente e investigadora en la Fundación Universitaria Juan N. Corpas y líder coordinadora en el programa Rehabilitación de base comunitaria a la persona con diagnóstico de trastorno mental (A. SALUDARTE). Bogotá, D.C., Colombia. ORCID: 0000-0001-5078-2053. Autor para correspondencia.

Correo electrónico: maritza.barroso@juanncorpas.edu.co

Enfermera, egresada de la Escuela de Enfermería de la FUJNC. Bogotá, D.C., Colombia.

Correo electrónico: jennifer-revelo@juanncorpas.edu.co

${ }^{3}$ Enfermera, egresada de la Escuela de Enfermería de la FUJNC. Bogotá, D.C., Colombia.

Correo electrónico:wendy-torres@juanncorpas.edu.co

^Enfermero, egresado de la Escuela de Enfermería de la FU JNC. Bogotá, D.C., Colombia.

Correo electrónico: holman-torres@juanncorpas.edu.co
} 


\title{
INTERNALIZED STIGMA IN PEOPLE WITH MENTAL DISORDER, RESIDENTS IN SHELTERED HOME WITH COMMUNITY-BASED REHABILITATION-FIRST SEMESTER 2019
}

\begin{abstract}
:
Stigma refers to attitudes and beliefs that lead people to reject avoid and fear those whom they perceive as different. The objective of the research is to determine the level of internalized stigma in a population of patients with Severe Mental Disorder (SMD) living a community-based rehabilitation process, during the period January-June 2019. The methodology is observational-descriptive study, quantitative approach. Two instruments were used: survey of sociodemographic characterization of the person with mental disorder and the application of the Internalized Stigma of Mental IIIness Scale (ISMI) (Tanabe et ál., 2016). The results show the four measurements allow verifying that there is a $62,5 \%$ perception of stigma and discrimination. At the same time, resistance to stigma is $70 \%$, showing that there may be a social adaptation capable of attenuating social stigma so that it does not progress to internalized stigma. The conclusion is that it is evident that there is a high perception of alienation, stigma and discrimination, not only in the social sphere, which is where rejection and isolation of the patient is most reflected, but also at the family, work and personal level, but thanks to the high resistance generated by the person around this environmental stimulus, internalized stigma is low.
\end{abstract}

Keywords: mental disorders, social stigma, health education, theory of mind, schizophrenia, social isolation. 


\section{Introducción}

El estigma social hacia las personas con una enfermedad mental ha sido ampliamente reportado, al ser uno de los colectivos que experimentan más estigma y más rechazo social (Ruiz et ál., 2012). Así mismo, la Declaración Universal de los Derechos Humanos ha desarrollado indicadores que ponen en evidencia la estigmatización de la que son víctimas las personas en condición de discapacidad psicosocial (World Health Organization [WHO], 2018). Las tres primeras causas de mortalidad a nivel mundial tienen que ver con la internalización del estigma en pacientes que padecen de TM grave y recurrente. La Asociación Americana de psiquiatría (APA) ha demostrado que el $85 \%$ de la población general ignora lo que es el TM; esto produce el estigma, que conduce a un comportamiento errático, excluyente e irresponsable, de manera que: a) ven las afecciones de salud mental como si fueran una sola, sin diferenciarlas, y a todos les Ilaman despectivamente locos, y b) tampoco diferencian la severidad, por ello ven un problema o un trastorno mental que es igual, sin tener en cuenta la intensidad y extensión de los síntomas, lo cual puede generar afectaciones mortales en pacientes diagnosticados con TM, quienes pueden recurrir al suicidio como medio de "salida". Incluso, se ha encontrado que las personas que padecen de TM son mayormente estigmatizadas que aquellas que padecen del VIH o enfermedades contagiosas (Ruiz et ál., 2012).

El estigma social tiene componentes emocionales, cognitivos y conductuales. En el aspecto cognitivo, la persona se percibe de forma negativa como peligrosa o incompetente; en cuanto a lo emocional, aparecen sentimientos de culpa, vergüenza o desconfianza, derivados en gran medida por la discriminación que reciben las personas con TM (Corrigan y Watson, 2002). El 50\% de la población adulta diagnosticada tardó mucho en pedir atención especializada, debido a la no aceptación de su diagnóstico o condición (Organización Mundial de la Salud [OMS], 2019). Tanto el plan de acción sobre salud mental 20132020 (OMS, 2013) como el plan de acción mundial para superar las brechas mhGAP (Organización Panamericana de la Salud [OPS], 2017) han desplegado todos sus recursos en el afán de atenuar las cifras que demuestran el escaso número de personas que son atendidas y, por tanto, diagnosticadas y tratadas, en comparación con las personas que lo requieren en Colombia. Según la encuesta nacional de salud mental (MinSalud y Colciencias, 2015), de los adultos entre dieciocho a cuarenta y cuatro años reciben atención solamente un 38,5\% y después de haberlo solicitado un promedio de 23,5 veces, lo que podría interpretarse dentro de las barreras actitudinales que evidencian estigma y discriminación. De allí la importancia de trabajar en pro de esta población e identificar cuál es el nivel de autoestigma para así generar respuestas efectivas, ya que es bien sabido que la detección temprana y la atención oportuna de cualquier problema de salud mejora el pronóstico y la severidad de sus síntomas. La principal derrota en el esfuerzo por compensar los trastornos mentales se evidencia en el comunicado de prensa de la OPS y la OMS (2014): "Más de 800000 personas mueren por suicidio cada año en el mundo -aproximadamente, una cada 40 segundos-, según el primer informe sobre prevención del suicidio de la Organización Mundial de la salud". En este sentido, se hace un Ilamado sobre el fracaso en la atención de los trastornos mentales según las cifras. El objetivo de esta investigación es determinar el nivel de estigma internalizado en una población de pacientes con TM, residentes en un hogar protegido con rehabilitación de base comunitaria durante el primer semestre de 2019.

\section{Metodología}

En el estudio observacional y descriptivo de enfoque cuantitativo, la población de estudio fueron pacientes residentes en un hogar protegido con rehabilitación de base comunitaria, diagnosticados con TM, específicamente aquellos con esquizofrenia paranoide. Se incluyeron en la muestra personas residentes en un hogar protegido con rehabilitación de base comunitaria, con una estancia mayor de un año; personas con diagnóstico de TM según definición del DSM-5 (Muñoz y Jaramillo, 2015), con una cronicidad de al menos dos años según historia clínica, y personas que aceptaron participar en el estudio, a través de la firma del consentimiento (paciente) y el asentimiento informado (familiar o responsable). Se excluyeron a dos personas con diagnóstico de TM 
por presentar síntomas refractarios, delirios y alucinaciones que impidieron un suficiente contacto con la realidad para sostener la entrevista.

\section{Técnicas de muestreo}

Dado que la población objeto del estudio está limitada a las personas diagnosticadas con TM, residentes de un hogar protegido con rehabilitación de base comunitaria, según su capacidad instalada, se realizó un muestreo no probabilístico, seleccionado de acuerdo al criterio del equipo profesional tratante y los casos de pacientes de mayor utilidad para el estudio.

\section{Técnicas de recolección de los datos}

En aras de desarrollar los objetivos específicos del estudio, se procedió a obtener los datos principales a través de las siguientes técnicas:

1. Encuesta de caracterización sociodemográfica de la persona con alteración mental (de construcción de los autores).

2. Aplicación de la escala de autoestigma de la enfermedad Internalized Stigma of Mental IIIness Scale (ISMI) (Tanabe et ál., 2016), la cual es un instrumento psicométrico de veintinueve ítems que evalúan el estigma internalizado a la persona con TM. Se realiza a partir de una escala tipo Likert que fluctúa entre muy de acuerdo hasta muy en desacuerdo (al puntuar de 1 a 4); en su totalidad, este instrumento integra tres subescalas, a saber: discriminación, divulgación y aspectos positivos de la enfermedad mental.

Es necesario tener claridad en tres conceptos que manejan esta escala:

El estigma se encuentra constituido de tres principales elementos: 1) estereotipo: creencia negativa que describe al individuo por poseer características que resultan inadecuadas o exageradas; 2) prejuicio: reacción emocional negativa resultado de los estereotipos, y 3) discriminación: conducta negativa resultado de los prejuicios. (Tanabe et ál., 2016)

Según los autores el estigma internalizado, este hace referencia al efecto que tiene sobre la persona, familia o colectivo este complejo estig- ma-discriminación, de manera que, al considerar cierto lo que dice la sociedad sobre sí mismo, esto se define como estigma internalizado. Este hace referencia a un comportamiento de exclusión o evasión que hace la persona al aislarse de lo que puede generarle estigma. El test mide también el logro de adaptación social como una resistencia al estigma, la cual se define como la capacidad que tiene una persona, familia o colectivo de filtrar estos mensajes y comportamientos al reemplazarlos por otros calificativos a criterio personal capaz de atenuar y defenderse de esa despiadada asignación (Paredes Márquez et ál., 2012).

Una vez obtenidos los datos, estos se procesaron y se analizaron por medio de un archivo de Excel, los cuales generaron medidas de tendencia central que dan cuenta de los resultados obtenidos.

\section{Aspectos éticos}

Para esta investigación, nos basamos en lo estipulado por la resolución 8430 de 1993, por la cual se establecen las normas científicas, técnicas y administrativas para la investigación en salud. Según los términos de esta resolución en su artículo 11, esta investigación estaría clasificada como investigación con riesgo mínimo. La resolución también hace especificaciones en el caso de pacientes con afecciones mentales. En su artículo 23 y 25 del capítulo III, hace referencia a que, para realizar investigaciones con menores de edad o discapacitados físicos o mentales, deberá obtenerse el consentimiento informado de quienes ejerzan la patria potestad o la representación legal del discapacitado (MinSalud, 1993). Tanto los residentes como los familiares dieron su consentimiento para aplicar la prueba, que no ofrece ningún riesgo para la salud mental de los sujetos de investigación.

\section{Resultados}

Hemos seleccionado los resultados demográficos que son marcadores de estigma social y que con mayor facilidad permiten que estos estereotipos se refuercen en la vida privada, para que el individuo sea permeable al estigma y no lo internalice. Seleccionamos las respuestas a la escala, que señalan con mejor precisión las conclusiones a las que llega el presente estudio con respecto 
a los tres aspectos relevantes: percepción de estigma, resistencia al estigma y estigma internalizado.

Figura 1. Género

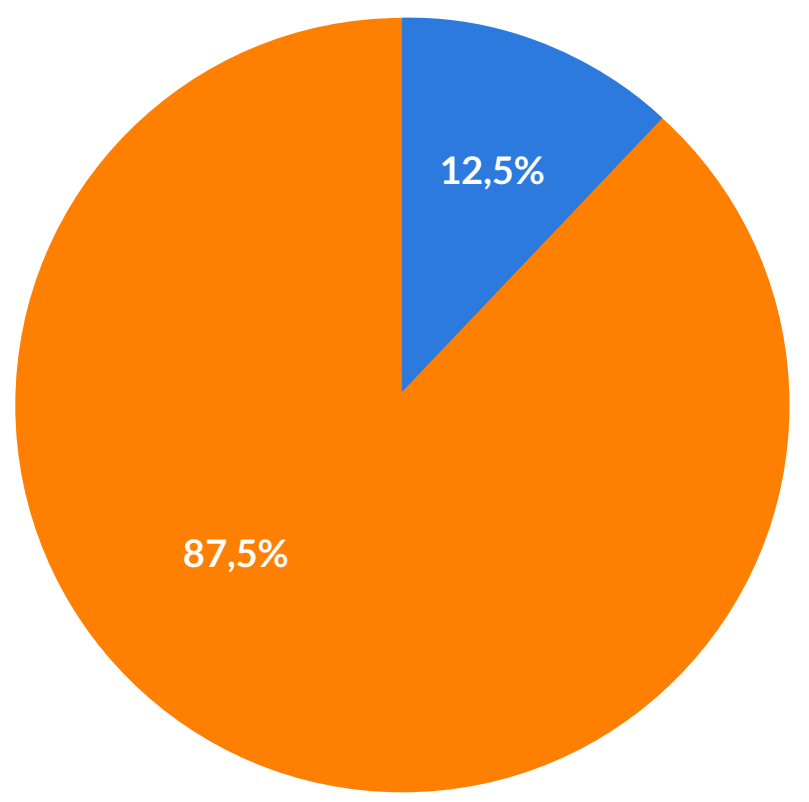

Femenino $n=1 \quad$ Masculino $n=7$

Fuente: elaboración propia.

Figura 3. Medición de percepción de estigma

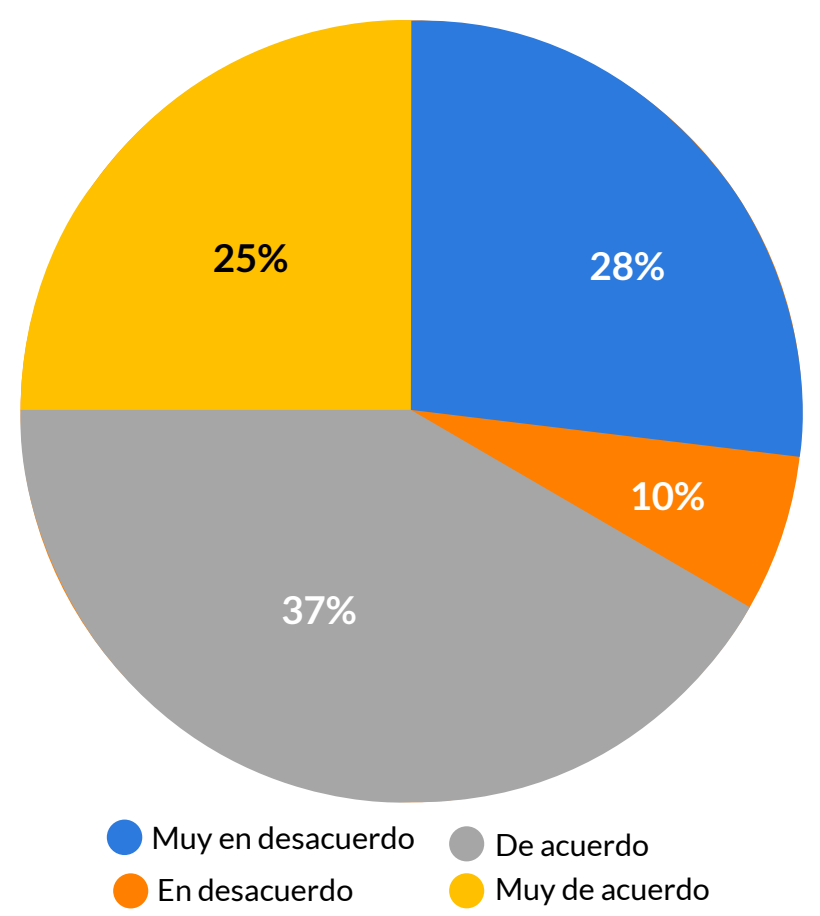

Fuente: elaboración propia.
A partir las figuras 1-4, llama la atencion que, según el género, 12,5\% son mujeres y el 87,5\% son hombres. Por su parte, en la valoración del

Figura 2. Ápgar familiar

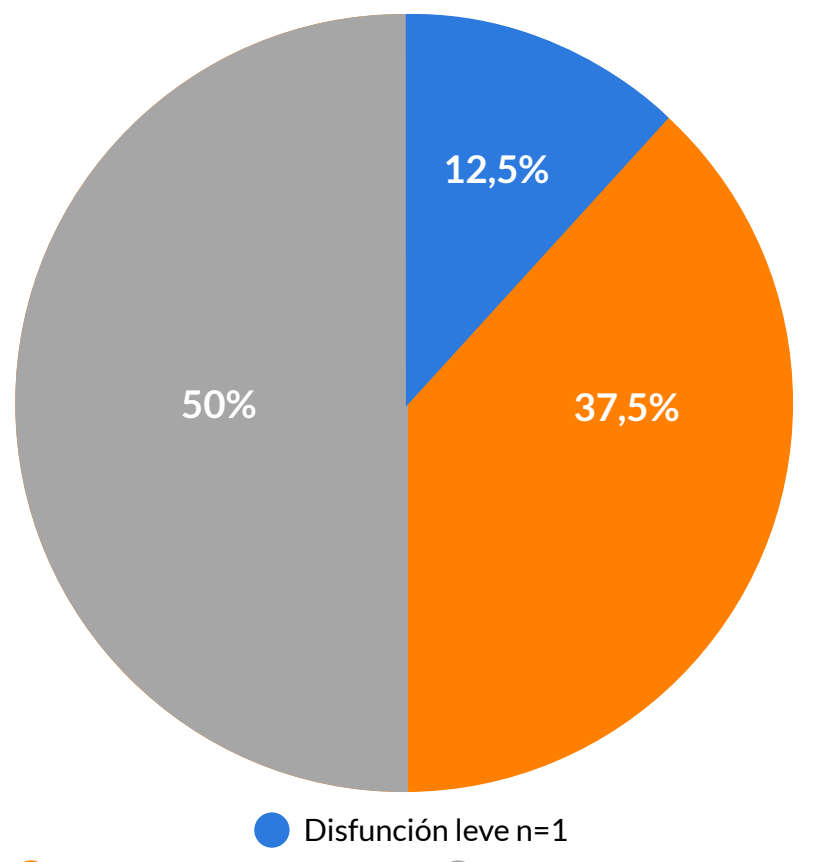

Disfunción moderada $\mathrm{n}=3$

Fuente: elaboración propia.

Figura 4. Resistencia al estigma

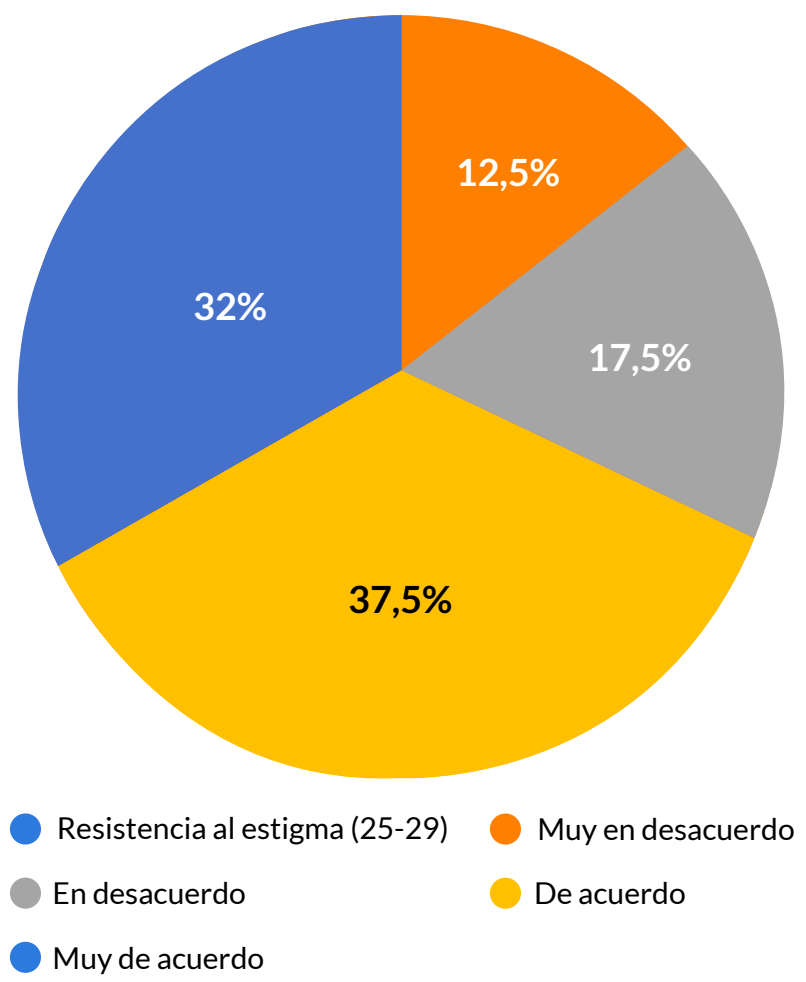

Fuente: elaboración propia. 
ápgar familiar, se encontraron disfunciones familiares severas en un 50\% de los casos. Así mismo, al analizar las respuestas de las preguntas 1 a la 24, a fin de identificar la percepcion que tienen las personas encuestadas (pacienes con diagnóstico de esquizofrenia paranoide) a los prejuicios y estereotipos sociales y estructurales, se encuentra un porcentaje del $62,5 \%$ en la perceción de estigma en su entorno familiar y social. La resistencia al estigma es de $70 \%$. Es probable que un efecto a favor de estos resultados sea el hecho de hallarse en una superficie de cuidado con marcado enfasis en la vida comunitaria y la inclusión social.

Este círculo consta de estereotipos sociales, estigma, discriminación, estigma internalizado, discriminación anticipada, temor de consultar, temor al tratamiento psiquiatrico, descompensación del trastorno y estado descompensado. Todo esto puede encaminarse a la tragedia del suicidio, a la perdida de la red familiar, al desprestigio social o se puede recibir protección y ser incluido en procesos comunitarios que, como en este caso, demuestra producir resistencias y romper este círculo que produce enormes sufrimientos a pacientes y familiares.

Cuando se logra control de síntomas, la persona puede tener calidad de vida, una familia y una red que le dé soporte y, entonces, el trastorno puede ser llevado con dignidad.

\section{Discusión}

Según el primer objetivo específico, al identificar las características sociodemográficas de la persona con diagnóstico de TM residente de un hogar protegido con rehabilitación de base comunitaria se observa el género de las personas encuestadas y se evidencia que la mayoría de los encuestados son hombres, al tener en cuenta que la enfermedad específica de estas personas es esquizofrenia. Es válido afirmar que estas cifras guardan relación respecto a lo que afirma la OMS, pues esta indica que más de veintiún millones de personas en el mundo padecen de esta enfermedad, de los cuales doce millones son hombres, mientras que nueve millones son mujeres, al ser además los hombres quienes desarrollan la enfermedad con mayor frecuencia a edades tempranas (Moreno et ál., 2016).

Por un lado, se encontró que el 50\% de la población pertenece a estrato social uno, cuatro de ellos afiliados al régimen contributivo y cuatro al régimen subsidiado. Esto último puede implicar que estén más vulnerables al estigma social e interiorizado, debido a que no cuentan con los recursos o medios necesarios para su manejo médico, lo que dificulta el apego al tratamiento instaurado y, por ende, genera más demoras en sus procesos de recuperación. Sin embargo, se rompe una barrera en cuanto a los determinantes sociales, en especial de las personas que pertenecen al régimen subsidiado: este tipo de régimen, lo que indica por norma general, es que la persona se encontraría en un posible estado de vulnerabilidad, dado que es probable que sus determinantes sociales estén afectados (Grandón Fernández et ál., 2016).

Por otro lado, se puede observar que el 100\% de la población de estudio presenta disfunción familiar, así: 50\%, disfunción familiar severa; el $37.5 \%$, disfunción moderada, y el 12.5\%, disfunción leve. Lo anterior quiere decir que en estas personas se evidencia otro dato relevante, que es el debilitamiento de las redes de apoyo de la familia en cuanto a las necesidades del paciente, lo que dificulta o entorpece que este obtenga herramientas adecuadas para afrontar o sobrellevar la enfermedad mental como se espera. Sin embargo, encontramos que estas personas cuentan con un aspecto favorable y es el hecho de que el centro donde viven cuenta con una ubicación en zonas residenciales de alto estrato social, como lo es el barrio Niza o Modelia. Del total de entrevistados, el 37,5\% habitan en el primero y, en el segundo, el otro 62,5\%, quienes cuentan con una infraestructura adecuada y con profesionales idóneos para favorecer su calidad de vida y la adherencia al tratamiento. Todo esto favorece significativamente el nivel de vulnerabilidad de estas personas en cuanto a los determinantes sociales, pues en concordancia con la OMS, existe un impacto en cuanto a la reducción de los factores que afectan o representan una barrera que desencadenan en la puerta de entrada al deterioro de la calidad de vida y las barreras 
en la atención sanitaria. La superficie de cuidado gestiona las rutas de atención con las respectivas EPS, al eliminar las barreras existentes para recibir la atención sanitaria (Grandón Fernández et ál., 2016).

Al tener en cuenta los determinantes sociales de la salud, encontramos que la mayoría de las personas participantes en esta encuesta no tienen educación superior e inclusive no culminaron su primaria, lo que puede ser un factor predominante para no entender o aceptar su enfermedad de manera enriquecedora. Esto facilita la existencia del estigma interiorizado, pues estas personas no cuentan con las herramientas intelectuales suficientes para comprender completamente las implicaciones personales y sociales que conlleva el diagnóstico de esquizofrenia. De acuerdo con Ruiz et ál. (2012), el ideal es que las personas tengan un nivel de educación adecuado, que permita comprender lo que es un TM y por ende permita que las personas con este se rodeen de lugares adecuados que favorezcan una óptima calidad de vida. Así como Katherine Colkaba lo menciona en su teoría del confort (este no es más que la respuesta que presentan los receptores de las intervenciones ofrecidas), la cual alude a la importancia que tienen los tres contextos que enfatizan el cuidado, en los cuales se incluyen el de aspectos ambientales, demográficos y el de los sujetos de cuidado (en el que hace un énfasis especial). Estos deben ser idóneos para obtener una respuesta favorable por parte de los receptores de cuidado, sin importar el tipo de enfermedad por el cual esté cursando la persona sujeta de cuidado (Merino-Martínez et ál., 2019).

Basados en el segundo objetivo específico, medir el estigma internalizado que tienen las personas que padecen TM con la escala Internalized Stigma of Mental IIIness Scale, se puede analizar la gráfica que agrupa la subcategoría denominada percepción del estigma, en la cual se encuentra el $62,5 \%$ de las personas que respondieron esta entrevista. Esto nos indica que existe una alta percepción de estigma, pues las respuestas de los entrevistados identifican que en algún momento se han visto enfrentados a estigma o discriminación familiar o social.
Sin embargo, se evidencia en el quinto ítem una resistencia al estigma del $70 \%$ del total de los encuestados. Este resultado se puede analizar desde la perspectiva de Naranjo Valentín et ál. (2017), quienes hacen referencia a la manera de reducir el estigma hacia los pacientes con TM. Al comparar los resultados de este estudio con el que realizamos en el hogar protegido con rehabilitación de base comunitaria, podemos identificar cómo el conocimiento y el incluir a la persona con TM a una vida normal con el resto de personas que no padecen esta enfermedad favorece la calidad de vida de la persona, así como la resistencia al estigma y la adherencia al tratamiento. Así como Naranjo Valentín et ál. (2017) consideraron la posibilidad de vincularlos al papel laboral y luego identificaron qué aspectos cambian en la sociedad y en el paciente diagnosticado después de desempeñar su papel como empleado, al obtener una mejor percepción por parte de los pacientes, nosotros encontramos que, a mayor percepción de estigma, en este caso el 62,5\%, mayor es la resistencia a este. En dicho caso, el $70 \%$, pues estas personas usan el conocimiento de su enfermedad y de la percepción social de esta no de forma que les afecte, sino como herramienta para afrontar su situación actual.

Sin embargo, en Colombia aún no hay datos específicos respecto al estigma de personas con TM. Es válido afirmar que la mayoría de la población encuestada, es decir, el 62,5\%, considera que no cumple con las características del estereotipo social que estigmatiza a la persona con TM, pero sí lo reconoce como un concepto negativo que tiene la sociedad de su condición. La evidencia de que el $70 \%$ de la población encuestada hace resistencia al estigma guarda relación directa con la alta percepción de estigma, que podría también estar relacionada con las condiciones socioeconómicas y de cuidado que reciben tanto en la ubicación de la vivienda como también el cuidado y tratamiento, en el contexto de la rehabilitación en base comunitaria que hace procesos de inclusión social.

Sabemos que los prestadores de servicios de salud también son invadidos por estos prejuicios sociales y a ello se deben las barreras de acceso a aquellos, lo que representa un obstáculo en la adherencia y permanencia en el tratamiento de 
las personas con TM, así como Grandón Fernández et ál. (2016) lo demuestran en su estudio. Por lo tanto, es muy probable que, de no haber una adecuada intervención por parte del equipo profesional, el nivel de resistencia sería menor el estigma social sería internalizado en mayor porcentaje entre las personas encuestadas.

Según la entrevista aplicada, las personas con trastorno mental no se consideran a sí mismas peligrosas ni incapaces de afrontar situaciones cotidianas, sin embargo, reconocen los ojos con los que la sociedad les observa. Como lo afirma Erwin Goffman (1963) en su publicación Estigma: la identidad deteriorada: el estigma es una etiqueta social, los procesos de estigmatización emergen desde la discrepancia entre una identidad social infringida y virtual, al hacer referencia a las características que debe tener una persona según la sociedad actual, así como unas características similares a la población en general. El estigma internalizado se refiere al estigma que siente cada persona. Livingston y Boyd (citados en Padín, 2012) lo definen como "un proceso subjetivo, imbuido en un contexto socio-cultural, que se caracteriza por sentimientos negativos (sobre sí mismo), comportamientos desadaptativos, transformación de la identidad o aplicación de estereotipos resultantes de una experiencia individual, percepción o anticipación de reacciones sociales negativas derivadas de su enfermedad mental".

\section{Conclusiones}

Según los resultados encontrados y en concordancia con el objetivo general de esta investigación, se concluye que la percepción de estigma de las personas con TM, residentes de un hogar protegido con rehabilitación de base comunitaria, está presente en el 62,5\% de la población total encuestada. Así mismo, que el $70 \%$ de las personas a quienes se les aplicó la escala hacen resistencia al estigma. En cuanto a las respuestas obtenidas, la alta percepción del estigma se relaciona con mejor aceptación de los síntomas, pues identificar los estereotipos que generan el estigma facilita tener conciencia de su enfermedad. Esto les permite adquirir herramientas que les favorezcan, adherirse al tratamiento, mejorar su autoestima, empoderarse y orientar sus recursos hacia la recuperación; por tanto, entendemos que esta resistencia ha protegido estos pacientes contra el estigma internalizado.

Finalmente, es importante difundir este resultado que demuestra la importancia de una rehabilitación basada en comunidad, que permite a las personas evidenciar su realidad y levantar barreras para evitar ser invadidos por calificativos devaluadores que afectan en todo sentido su realización de vida digna.

\section{Referencias Bibliográficas}

1. Corrigan, P. W., \& Watson, A. C. (2002). Understanding the impact of stigma on people with mental illness. World psychiatry: official journal of the World Psychiatric Association (WPA), 1(1), 16-20.

2. Fernando Muñoz, L., \& Jaramillo, L. E. (2015). DSM-5: ¿cambios significativos? Revista de la Asociación Española de Neuropsiquiatría, 35(125), 111-121. https://dx.doi.org/10.4321/S021157352015000100008

3. Goffman, E. (1963). Stigma: Notes on the management of spoiled identity. Prentice-Hall.

4. Grandón Fernández, P., Saldivia Bórquez, S., Cova Solar, F., Bustos, C., \& Turra Chávez, V. (2016). Análisis psicométrico y adaptación de la escala de actitudes comunitarias hacia la enfermedad mental (CAMI) en una muestra chilena. Universitas Psychologica, 15(2), 153-162. https://doi. org/10.11144/Javeriana.upsy15-2.apae 
5. Merino-Martínez, R. M., Morillo-Gallego, N., Sánchez-Cabezas, A. M., Gómez-López, V. E., \& Crespo-Montero, R. (2019). Relación entre la calidad de vida relacionada con la salud y la ansiedad/ depresión en pacientes en hemodiálisis crónica. Enfermería Nefrológica, 22(3), 274-283. https:// dx.doi.org/10.4321/s2254-28842019000300006

6. Ministerio de Salud (MinSalud). (1993). Resolución 8430 de 1993. Gobierno de Colombia. https://www.minsalud.gov.co/sites/rid/Lists/BibliotecaDigital/RIDE/DE/DIJ/RESOLUCION8430-DE-1993.PDF

7. Ministerio de Salud (MinSalud) y Colciencias. (2015). Encuesta Nacional de salud mental-2015. Gobierno de Colombia, Colciencias. http://www.odc.gov.co/Portals/1/publicaciones/pdf/consumo/estudios/nacionales/C0031102015-salud_mental_tomol.pdf.

8. Moreno Fergusson, M. E., Muñoz de Rodríguez, L., Alemán Parra, C. M., Ávila Martín, L. A., Badillo Romero, K. T., \& Barajas Lizarazo, M. A. (2016). De la teoría de enfermería a la práctica experiencias con proyectos de gestión del cuidado (1. ed.). Universidad de la Sabana.

9. Naranjo Valentín, R., Cobo Martínez, F., Rebolleda-Gil, C., \& Gonzáles Fraile, E. (2017). Centro de rehabilitación laboral y trastorno mental grave: experiencia y seguimiento de usuarios en la comunidad de Madrid. Comunicaciones Libres Sujetos a lo Social, 1.

10.OrganizaciónMundialdelaSalud(OMS)(2013).Plandeacciónsobresaludmental2013-2020.[online] Disponible desde: https://apps.who.int/iris/bitstream/handle/10665/97488/9789243506029_ spa.pdf [Acceso el 27 Septiembre 2021].

11. Organización Mundial de la Salud (OMS). Desórdenes mentales. (2019). OMS.

12. Organización Panamericana de la Salud (OPS) (2017). Guía de intervención MHGAP para los trastornos mentales, neurológicos y por consumo de sustancias en el nivel de atención de salud no especializada. Versión 2.0. OPS. Https://Iris.Paho.Org/Handle/10665.2/34071

13. Organización Panamericana de la Salud (OPS) y Organización Mundial de la Salud (OMS). (2014). Comunicado de prensa. Más de 800000 personas mueren por suicidio cada año en el mundo, según un nuevo informe de la OMS. Salud mental, 37(5), 449-450. Recuperado el 27 de septiembre de 2021 de http://www.scielo.org.mx/scielo.php?script=sci_arttext\&pi$d=S 0185-33252014000500012 \&$ Ing=es\&tlng=es.

14. Padín, G. A. (2012). Esquizofrenia. Cuadernos del Tomás, (4), 151-172. Recuperado el 3 de octubre de 2018 de https://dialnet.unirioja.es/servlet/articulo?codigo=4018442

15. Paredes Márquez, H. M., Jiménez Trejo, G., \& Vázquez Estupiñán, M. F. (2012). Medición edición del estigma internalizado y su relación con la adaptación social en pacientes psiquiátricos. Vertientes Revista Especializada en Ciencias de la Salud, 15(2), 88-92.

https://doi.org/https://www.medigraphic.com/pdfs/vertientes/vre-2012/vre122d.pdf

16. Ruiz, M. Á., Montes, J. M., Lauffer, J. C., Álvarez, C., Mauriño, J., \& de Dios Perrino, C. (2012). Opinions and beliefs of the Spanish population on serious mental illnesses (schizophrenia and bipolar disorder). Revista de Psiquiatría y Salud Mental (English Edition), 5(2), 98-106.

https://doi.org/10.1016/j.rpsmen.2012.01.003 
17. Tanabe, Y., Hayashi, K., \& Ideno, Y. (2016). The Internalized Stigma of Mental IIIness (ISMI) scale: validation of the Japanese version. BMC Psychiatry, 16, 116. https://doi.org/10.1186/s12888016-0825-6

18. World Health Organization (WHO). (2018). Salud mental: fortalecer nuestra respuesta. Who.int. WHO. https://www.who.int/es/news-room/fact-sheets/detail/mental-health-strengthening-ourresponse 